\title{
Fast aperiodic variability in the black hole binary GRS 1915+105: the timing signature of relativistic ejection events
}

\section{Paolo Soleri*}

Astronomical Institute "A. Pannekoek", University of Amsterdam and Center for High-Energy Astrophysics, Kruislaan 403, NL-1098 SJ, Amsterdam, The Netherlands

E-mail: psoleri@science.uva.nl

\section{Tomaso Belloni}

INAF-Brera Astronomical Observatory, Via E. Bianchi 46, I-23807, Merate, Italy

E-mail: tomaso.belloni@brera.inaf.it

\section{Piergiorgio Casella}

Astronomical Institute "A. Pannekoek", University of Amsterdam and Center for High-Energy Astrophysics, Kruislaan 403, NL-1098 SJ, Amsterdam, The Netherlands

E-mail: casella@science.uva.nl

\begin{abstract}
We present X-ray observations of the Black-Hole Binary GRS 1915+105 made with the RXTE (Rossi X-ray Timing Explorer). We concentrated on timing analysis of the strong variability focusing on its aperiodic variability on short $(<1 \mathrm{~s})$ time scales. In the power density spectra, we found a feature which is seen in many transient systems, but until now was not detected in GRS 1915+105 due to its elusiveness. As this feature has been associated to the collimation and emission of superluminal relativistic jets visible in the radio band, its presence on the prototypical galactic jet source strengthens this connection. Since its discovery in 1992, GRS 1915+105 was considered a peculiar source. Our results suggest that its general behaviour is similar to that of other black-hole binaries, but its state-transitions are much faster and difficult to analyze.
\end{abstract}

VI Microquasar Workshop: Microquasars and Beyond

September 18-22 2006

Società del Casino, Como, Italy

${ }^{*}$ Speaker. 


\section{Introduction}

Systematic variations in the energy spectra and intensity of transient Black Hole Candidates (BHCs) have been recently identified in terms of the pattern described in an X-ray Hardnessintensity diagram (HID, see [1], [9]). Four main bright states (in addition to the quiescent state) have been found to correspond to different branches/areas of a square-like HID pattern. In this framework much importance is given to the intermediate states (called Hard intermediate state, HIMS, and Soft intermediate State, SIMS), and to the transitions between them, identified from the behaviour in several bands of the electromagnetic spectrum (from radio to hard X-ray, see [8]) and from the timing properties of the X-ray light curve.

Low-frequency Quasi-Periodic Oscillations (LFQPOs) with frequencies ranging from a few $\mathrm{mHz}$ to tens of $\mathrm{Hz}$ are a common feature in black hole candidates and have been observed in many galactic BHCs since the '70s (see [17], [11] and references therein). Three main types of LFQPOs, dubbed Type-A, -B and -C respectively, were originally identified in the light curve of XTE J1550-564 ([18], [16]) and are seen in several sources (see [4] and references therein). In the context of the state classification outlined above, it is possible to ascribe the three LFQPOs to different spectral conditions (see [1], [9]). The type-C QPO is associated to the (radio loud) HIMS and to the low/hard state. It is a common QPO seen in almost all BHCs with a variable centroid frequency correlated with the count rate, a high fractional variability and a high coherence $(Q=v / F W H M \sim 10)$. The type-B QPO has been seen in few systems (see [4]); it is a transient oscillation associated to the spectral transition from the (radio loud) HIMS to the (radio quiet) SIMS and its features are a $\sim$ constant centroid frequency, lower fractional variability and $Q$ than the type-C. Some authors ([8], [3]) suggested that these spectral transitions are in turn associated to the emission and collimation of relativistic radio jets. These jets are seen in a number of sources (GRS 1915+105, XTE J1550-564, GX 339-4, XTE J1859+226, etc.) but not in all of these sources we could resolve the spectral transition to see the transient QPO. The spectral properties connected to type-A QPO are similar to those introduced for the type-B. This QPO has been seen in few systems (see [4]), and is broader, weaker and less coherent than the type-B QPO.

GRS 1915+105 was discovered in 1992 with the GRANAT/WATCH ([5], [6]). It is the first galactc source observed to have apparently superluminal relativistc radio jets ([14]), interpreted as ejection of ultrarelativistc plasma. Its radio variability was discovered to correlate to the hard X-ray flux ([13]).

The rich phenomenology of this source was described (see [2]) in terms of spectral transitions between three basic states, A, B and C (not to be confused with the names of the LFQPOs), that give rise to more than a dozen variability classes. The non-standard behaviour of GRS 1915+105 was interpreted as that of a source that spends all its time in the Intermediate States, never reaching the LS or the quiescence ([7]). Until now all LFQPOs observed from this system can be classified as type-C QPOs; altough GRS 1915+105 makes a large number of fast state transitions, which have been positively associated to radio activity and jet ejection, no type-B QPO has been observed to date.

Here we present the discovery with RXTE of the type-B QPO in the X-ray light curve of GRS $1915+105$. The QPO was present during fast spectral transitions that can be identified with the HIMS to SIMS transition observed in other BHCs. A complete paper with a detailed description 


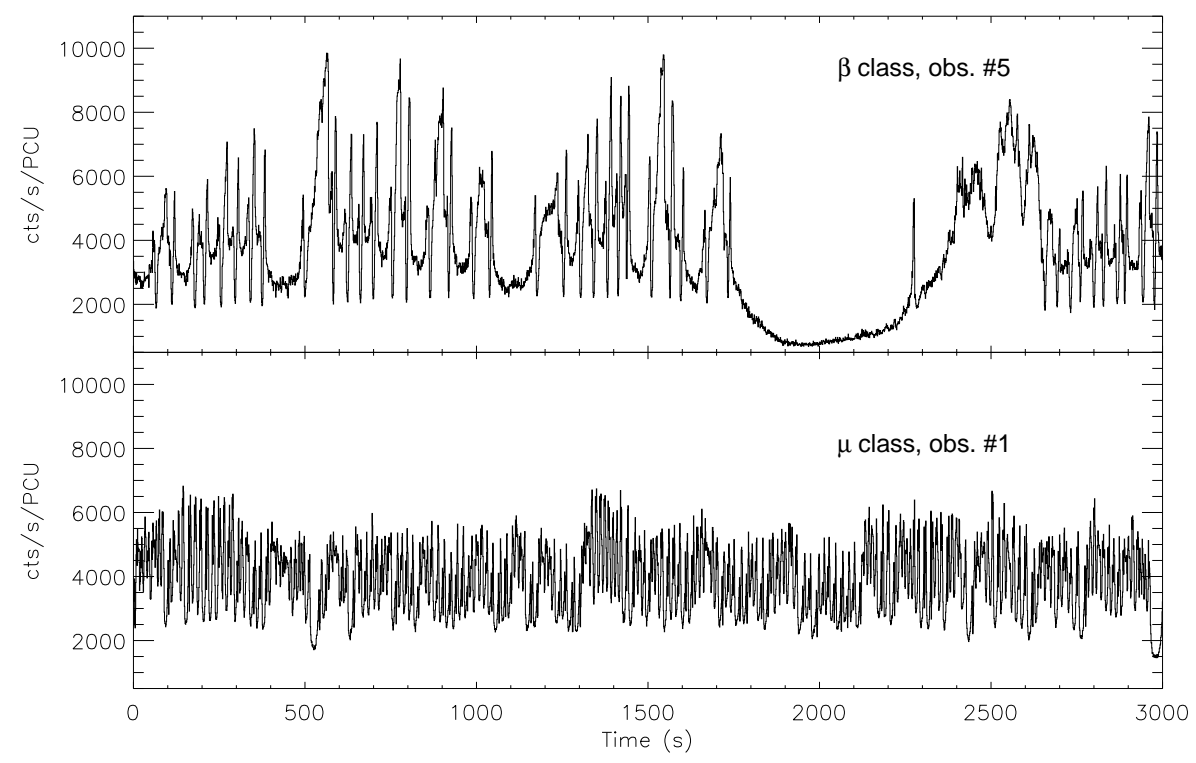

Figure 1: 2-13 keV light curves for the two variability classes examined here. Top panel: observation from 1997 Dec 17; bottom panel: observation from 1996 Sep 09. Bin size is 1 second.

of the data analysis and the results is in preparation.

\section{Observations and data analysis}

We analyzed five RXTE/PCA observations collected between 1996 September 22 and 1997 December 17. We chose observations belonging to variability classes $\mu$ and $\beta$ (according to the classification presented in [2], see Fig. 1 for two examples of light curves) because they correspond to intervals when frequent fast transitions among the three spectral states are observed. The transitions being fast, the type-B QPO would not be detected for a single transition, but adding a large number of data corresponding to the same transition, the signal could become detectable. For each observation we produced light curves in the 2-13 keV energy range and two hardness ratios $(\mathrm{HR} 1(5-13 \mathrm{keV}) /(2-13 \mathrm{keV})$; HR2 $(13-38 \mathrm{keV}) /(2-5 \mathrm{keV}))$; the bin size was 2 seconds for all these curves. Given the high count rates, we did not subtract the background. In addition, for each 2-second time interval, we accumulated a power density spectrum (PDS) in the 2-5 keV, 5-13 keV and 13-38 keV energy ranges with a time resolution of 1/128 sec. We also created 3-dimensional hardness-hardness-intensity diagram, where the source follows a regular path and we averaged the PDS corresponding to different regions in this diagram.

For a detailed description of all the data analysis see Soleri, Belloni, Casella, to be submitted.

\section{Results}

An example of 3D HHID diagram is shown by the two-dimensional projections in Fig. 2. The regular path followed by the source is marked by arrows. Starting from the region of the HHID with highest HR2 (right side of both the diagrams) we averaged power density spectra on small number of points $(\sim 300)$, following the source path, for all the observations. We found that in this 


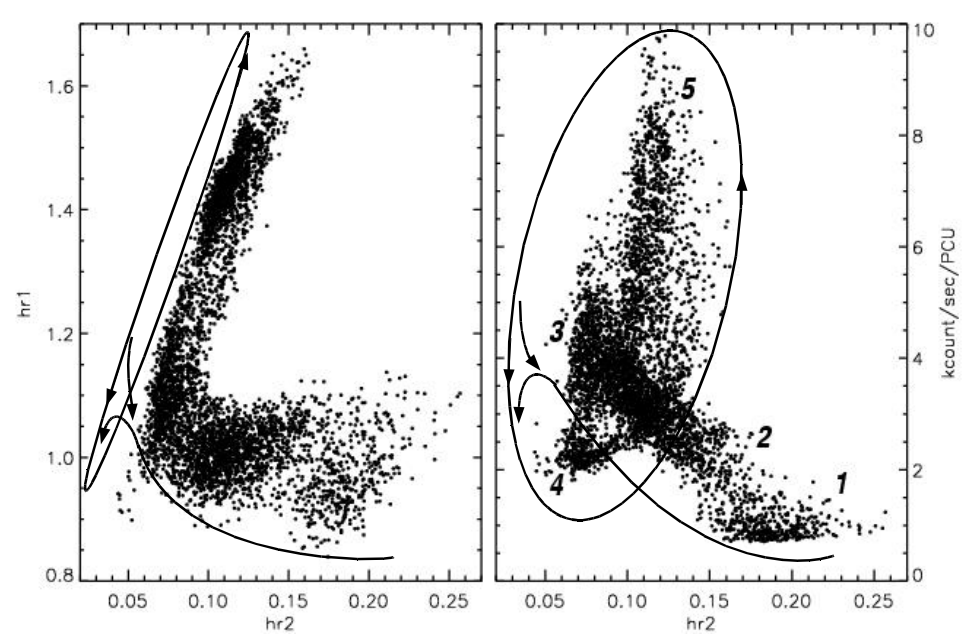

Figure 2: Two-dimensional projections of the hardness-hardness-intensity diagram (HHID) for one analyzed observation. Left side panels: color-color diagram; right side panels: hardness-intensity diagram. Definitions for the colors can be found in $\$ 2$, the count rate is the $2-13 \mathrm{keV}$ energy band. Arrows indicate the source regular and repetitive movement. Numbers correspond to the regions discussed in the text (see Fig. 3).

\begin{tabular}{cccccc}
\hline \hline & \multicolumn{5}{c}{ Regions in figure 3 } \\
Observation $\mathrm{N}^{\circ}$ & 1 & 2 & 3 & 4 & 5 \\
\hline 1 & - & $\mathrm{C}$ & $\mathrm{C}, \mathrm{B}$ & $\mathrm{B}$ & No QPOs \\
2 & Bump - C & C & C, B & No QPOs & No QPOs \\
3 & Bump - C & C & C, B & No QPOs & No QPOs \\
4 & - & C & C, B & B & No QPOs \\
5 & Bump - C & C & C, B & B & No QPOs \\
\hline
\end{tabular}

Table 1: QPO types detected in the five regions for all considered observations. Region 1 is not present in observations \# 2 and 3.

way it is possible to identify five average behaviours of the PDS, independently from the analyzed observation. These five behaviours correspond to five separated regions of the source path in the HHID that are shown in the left and central panels of Figure 3.

We describe the average PDS behaviour of these macro-regions in Tab. 1: from this table it is clear that we can use the same macro-region classification for different observations. As described in $\S 2$, we considered observations belonging to variability classes $\mu$ and $\beta$. These observations have very similar light curves and HHID, the only difference being that in class $\beta$ there are long hard intervals (the smooth stretch in the top panel of Fig. 1), which correspond to region 1 (Orange) and to the hardest part of region 2 (Green) in the HHID. Since we do not concentrate on the harder intervals, for our purposes these two classes can be treated as one.

We now describe in detail the power density spectra behaviour in all the identified regions:

1. starting from region 1 , we see a peak, increasing in frequency and in quality factor $Q$ (from a $Q<2$ for a centroid frequency $\sim 2 \mathrm{~Hz}$ to a $Q>2$ for higher centroid frequency) and decreasing in fractional $r m s$ amplitude moving towards region 2. Its fractional $r m s$ is higher 

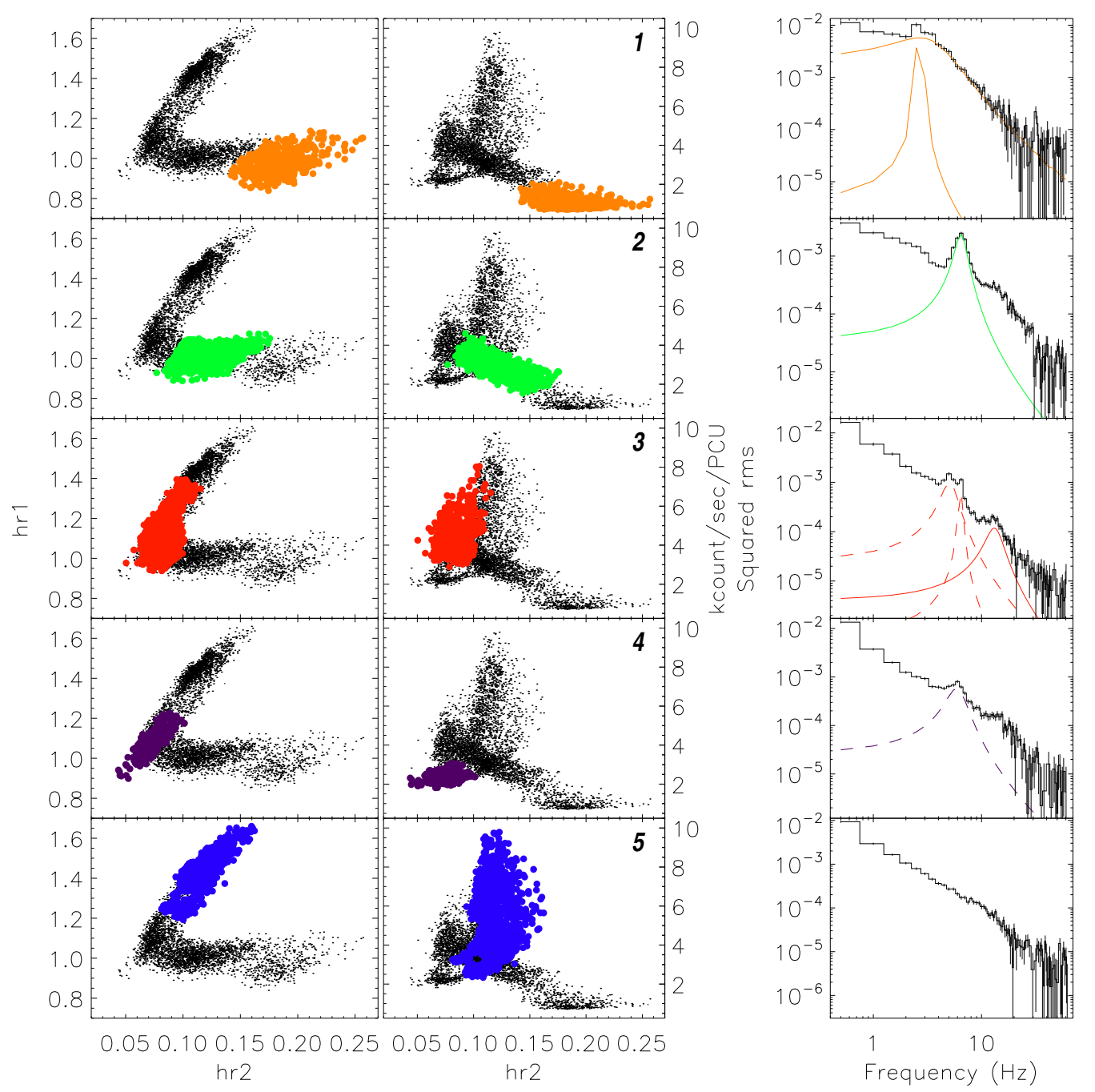

Figure 3: Two-dimensional projections of the hardness-hardness-intensity diagram for observation \#5 and typical power density spectra for each of the five regions. Left side panel: color-color diagrams; central panels: hardness-intensity diagrams. Definitions for hardness ratios can be found in $\S 2$, the count rate is the 2-13 keV energy band. Colored circles indicate the points corresponding to each region. Right side panels: power density spectra averaged through the points in the region in the $5-13 \mathrm{keV}$ energy band. With dashed lines we plot the Lorentzians used to fit type-B QPOs, with continuous lines the Lorentzians used to fit type-C QPOs.

in the hardest band (13-38 keV);

2. moving within region 2 towards region 3 , the peak continues this trend: increasing centroid frequency and decreasing fractional $r m s$ with the same energy dependence;

3. within region 3 , the peak still increases in frequency and decreases in fractional $r m s$, to disappear when approaching region 4 , corresponding to a frequency of $\sim 15 \mathrm{~Hz}$. At variance with what observed in previous regions, an additional peak appears. Its properties are 


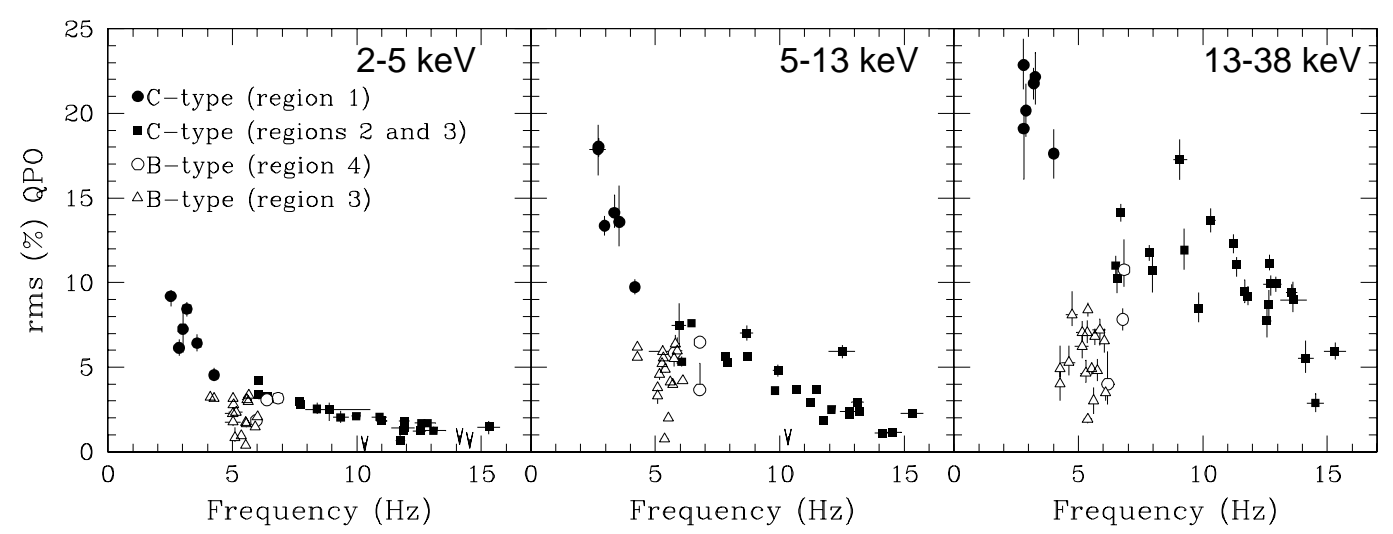

Figure 4: Fractional rms of the detected QPOs in all observations as function of their frequency for three energy bands. Error bars are estimated at $1 \sigma$ confidence level. Arrows indicate $3 \sigma$ upper limits for type-C QPOs only. Filled symbols indicate type-C QPOs, open symbols type-B. Left panel: energy band 2-5 keV (PCA channels 0-13). Central panel: energy band 5-13 keV (PCA channels 14-35). Right panel: energy band 13-38 keV (PCA channels 36-103).

markedly different from those of the QPO described above: it shows a much less variable frequency (from 4 to $6 \mathrm{~Hz}$ ) and a fractional rms independent of its centroid frequency. Its energy dependence is the same as that observed for the other peak. Averaging power spectra corresponding only to points close to the boundary to region 4 , only this second peak is visible.

4. in region 4, in some but not all of the observations (see table 1) a peak centered on a frequency between $\sim 6$ and $7 \mathrm{~Hz}$ is detected. Its $r m s$ amplitude value is similar to that of second peak of region 3 , with the same energy dependence.

5. in region 5, no QPOs are detected.

We will refer to the peak with centroid frequency ranging from $\sim 2$ up to $15 \mathrm{~Hz}$ as type-C QPO and to the peak with $4-7 \mathrm{~Hz}$ frequency detected in regions 3 and 4 as type-B QPO. This identification will be motivated below. A plot of the fractional rms amplitude of all the detected peaks as a function of their centroid frequency, for each of three energy bands analyzed, helps to clearly discriminate between the two types of QPO (see Fig. 4 ). The figures contain the data for all detections. If for one observation a significant QPO was detected only in one band, we repeated the fit fixing centroid and FWHM of the peak and obtained its fractional rms. Type-C QPOs are indicated with filled symbols, type-B QPOs with open symbols. It is evident that the two QPOs correspond to different behaviours in these diagrams. Type-B QPOs cluster in a small region of the diagrams, while type-C QPOs follow a trend.

The points with large errors in frequency correspond to detections where, due to the changing frequency of the type-C QPO, we need two Lorentzian components to obtain a satisfactory fit. For these points, we plot the average of the two centroid frequencies.

From figure 3 we can relate the presence of the two QPO-types to different zones of the source path in the HHID but we do not have any detailed information about the time evolution of the 


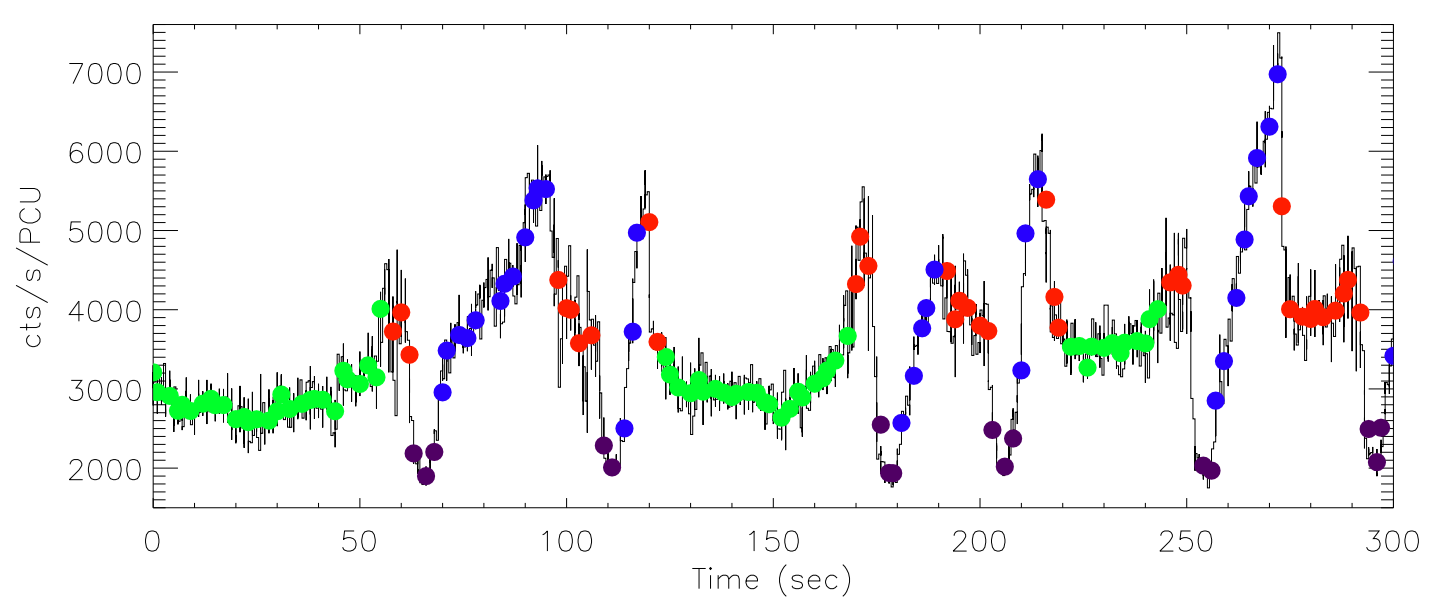

Figure 5: 300-second light curve (2-13 keV energy band) for observation \# 5. Bin size is $0.125 \mathrm{sec}$. Every colored point is averaged on 2 seconds. Different colors correspond to different regions in the HHID in Figure 3: Green = region 2; Red = region 3, Purple $=$ region 4; Blue $=$ region 5.

source position in that diagram. We know that $\mu$ and $\beta$ class are characterized by fast variability and frequent spectral transitions but we do not know precisely between which spectral states the transitions happen and when. A light curve where parts corresponding to different regions in the HHID are colored with the same colors used in Figure 3 can help us to clarify this point (see Fig 5). We chose a 300-second interval representative of the source behaviour. As mentioned above, we do not consider region 1 for this analysis. If, using the state classification presented in [2], we make the identifications Green $=\mathrm{C}$-state, Purple $=\mathrm{A}$-state, $\mathrm{Blue}=\mathrm{B}$-state and we consider the Red as a Transition (T) state, we have the following state sequences: CTAB TAB BTC.

\section{Conclusions}

We analyzed five RXTE/PCA observations and detected in all of them peaks that we identify with two different QPO-types: type-C and type-B QPO. This is the first detection of a type-B QPO in GRS $1915+105$. In all the five observations we detected the type-B together with the type-C, in two of them we also detected the type-B alone (see Tab. 1). We could not correlate the presence of the type-B in the fourth (Purple) region of Fig. 3 neither with the selected variability class nor with the count rate or the spectral hardness.

To date, a type-B QPO has been detected in few systems, associated to spectral transitions from HIMS to SIMS. In GRS $1915+105$ is well known the association between the X-ray and the radio activity (see [15], [12] and [7] for a review), therefore the non-detection of a type-B QPO was rather interesting, as alreday pointed out in [1]. Our detection of the type-B QPO in GRS 1915+105 strenghtens two points:

1. GRS $1915+105$ behaves like all other radio-loud BHCs: the type-B appears in correspondence of spectral transition from the B-state to the A-state (Transition, Red) and when the source is in the A-state (Purple). In the light curve in Fig. 5 we see state oscillations CTAB 
TAB TAB that we can identify as fast passages between the HIMS and the SIMS of the other BHCs ([8], [3]). The type-B appears also in correspondence of the transition from the B-state to the C-state (Blue-Red-Green in Fig. 5), so not in an oscillation event but in a transition from a soft to a hard state.

2. the association between type-B QPO, spectral transitions and radio jets emission. If we consider the type-B QPO as the signature radio jet emission, we have this "signature" also in the prototypical galactic jet source.

Klein-Wolt et al. (2002, see [10]) compared 101 simultaneous Ryle Telescope radio and RXTE X-ray observations of GRS $1915+105$ : they found that $\beta$ class observations are associated with strong radio oscillations while $\mu$ observations are positively associated to weak radio activity without oscillations. Unluckily, they did not analyze any of the observations from our data set so we can not verify directly GRS 1915+105 radio behaviour while the type-B QPO was present.

\section{References}

[1] T. Belloni, J. Homan, P. Casella, M. van der Klis, E. Nespoli, W. H. G. Lewin, J. M. Miller, and M. Méndez, The evolution of the timing properties of the black-hole transient GX 339-4 during its 2002/2003 outburst, A\&A 440, 207-222 (2005) [astro-ph/0 504577 ].

[2] T. Belloni, M. Klein-Wolt, M. Méndez, M. van der Klis, and J. van Paradijs, A model-independent analysis of the variability of GRS 1915+105, A\&A 355, 271-290 (2000) [astro-ph/0 001103 ].

[3] P. Casella, T. Belloni, J. Homan, and L. Stella, A study of the low-frequency quasi-periodic oscillations in the X-ray light curves of the black hole candidate XTE J1859+226, A\&A 426, 587-600 (2004) [astro-ph/ 0407262 ].

[4] P. Casella, T. Belloni, and L. Stella, The ABC of Low-Frequency Quasi-periodic Oscillations in Black Hole Candidates: Analogies with Z Sources, ApJ 629, 403-407 (2005) [astro-ph / 0504318 ].

[5] A. J. Castro-Tirado, S. Brandt, and N. Lund, GRS 1915+105, IAU Circ. 5590, 2-+ (1992).

[6] A. J. Castro-Tirado, S. Brandt, N. Lund, I. Lapshov, R. A. Sunyaev, A. A. Shlyapnikov, S. Guziy, and E. P. Pavlenko, Discovery and observations by watch of the X-ray transient GRS 1915+105, ApJS 92, 469-472 (1994).

[7] R. Fender and T. Belloni, GRS 1915+105 and the Disc-Jet Coupling in Accreting Black Hole Systems, ARA\&A 42, 317-364 (2004) [astro-ph/ 0406483 ].

[8] R. P. Fender, T. M. Belloni, and E. Gallo, Towards a unified model for black hole X-ray binary jets, MNRAS 355, 1105-1118(2004) [astro-ph/0409360].

[9] J. Homan, M. Buxton, S. Markoff, C. D. Bailyn, E. Nespoli, and T. Belloni, Multiwavelength Observations of the 2002 Outburst of GX 339-4: Two Patterns of X-Ray-Optical/Near-Infrared Behavior, ApJ 624, 295-306 (2005) [astro-ph/0501349].

[10] M. Klein-Wolt, R. P. Fender, G. G. Pooley, T. Belloni, S. Migliari, E. H. Morgan, and M. van der Klis, Hard X-ray states and radio emission in GRS 1915+105, MNRAS 331, 745-764 (2002) [astro-ph/0112044]. 
[11] J. E. McClintock and R. A. Remillard, Black hole binaries, pp. 157-213, Compact stellar X-ray sources. Edited by Walter Lewin Michiel van der Klis. Cambridge Astrophysics Series, No. 39. Cambridge, UK: Cambridge University Press, ISBN 978-0-521-82659-4, ISBN 0-521-82659-4, DOI: 10.2277/0521826594, 2006, p. 157 - 213, April 2006.

[12] I. F. Mirabel, V. Dhawan, S. Chaty, L. F. Rodriguez, J. Marti, C. R. Robinson, J. Swank, and T. Geballe, Accretion instabilities and jet formation in GRS 1915+105, A\&A 330, L9-L12 (1998) [astro-ph/9711097].

[13] I. F. Mirabel, P. A. Duc, P. A. Rodriguez, R. Teyssier, J. Paul, A. Claret, M. Auriere, D. Golombek, and J. Marti, The radio/infrared counterpart of the hard X-ray transient in Aquila: A possible source of repeated soft gamma-ray bursts, A\&A 282, L17-L20 (1994).

[14] I. F. Mirabel and L. F. Rodriguez, A Superluminal Source in the Galaxy, Nature 371, 46-+ (1994).

[15] G. G. Pooley and R. P. Fender, The variable radio emission from GRS 1915+105, MNRAS 292, 925-+ (1997) [astro-ph/9708171].

[16] R. A. Remillard, G. J. Sobczak, M. P. Muno, and J. E. McClintock, Characterizing the Quasi-periodic Oscillation Behavior of the X-Ray Nova XTE J1550-564, ApJ 564, 962-973 (2002) [astro-ph/0105508].

[17] M. van der Klis, Rapid X-ray Variability, pp. 39-112, Compact stellar X-ray sources. Edited by Walter Lewin Michiel van der Klis. Cambridge Astrophysics Series, No. 39. Cambridge, UK: Cambridge University Press, ISBN 978-0-521-82659-4, ISBN 0-521-82659-4, DOI: 10.2277/0521826594, 2006, p. 39 - 112, April 2006.

[18] R. Wijnands, J. Homan, and M. van der Klis, The Complex Phase-Lag Behavior of the 3-12 HZ Quasi-Periodic Oscillations during the Very High State of XTE J1550-564, ApJ 526, L33-L36 (1999) [astro-ph/9909515]. 